\title{
Bulk thermodynamics and charge fluctuations at non-vanishing baryon density
}

\author{
Chuan Miao and Christian Schmidt* (RBC-Bielefeld Collaboration) \\ Falkutät für Physik, Universität Bielefeld, D-33615 Bielefeld, Germany \\ Brookhaven National Laboratory, Upton, NY 11973, USA \\ E-mail: chuan@physik.uni-bielefeld.de, cschmidt@quark.phy.bnl.gvo
}

\begin{abstract}
We present results on bulk thermodynamic quantities as well as net baryon number, strangeness and electric charge fluctuations in QCD at non-zero density and temperature obtained from lattice calculations with almost physical quark masses for two values of the lattice cut-off $a T=1 / 4$ and $1 / 6$. We show that with our improved p4fa3-action the cut-off effects are under control when using lattices with a temporal extent of 6 or larger and that the contribution to the equation of state, which is due to a finite chemical potential is small for $\mu_{q} / T<1$. Moreover, at vanishing chemical potential, i.e. under conditions almost realized at RHIC and the LHC, quartic fluctuations of net baryon number and strangeness are large in a narrow temperature interval characterizing the transition region from the low to high temperature phase. At non-zero baryon number density, strangeness fluctuations are enhanced and correlated to fluctuations of the net baryon number. If strangeness is furthermore forced to vanish, as it may be the case in systems created in heavy ion collisions, strangeness fluctuations are significantly smaller than baryon number fluctuations.
\end{abstract}

The XXV International Symposium on Lattice Field Theory

July 30-4 August 2007

Regensburg, Germany

\footnotetext{
* Speakers.
} 


\section{Introduction}

Heavy-ion collision experiments at RHIC and LHC lead to thermalized dense matter at small but non-zero baryon density, or equivalently chemical potential. Therefore it is necessary to study the bulk thermodynamics of QCD at finite chemical potentials. In this work, we use the Taylor expansion method [1] to study the equation of state, the number density and fluctuations of various quantum numbers on the lattice. We study $2+1$ flavor QCD with tree level Symanzik-improved gauge action and $\mathrm{p} 4$ fat 3 -improved staggered fermion action [3]. The simulations are carried out on $16^{3} \times 4$ and $24^{3} \times 6$ lattices on a line of constant physics with almost physical quark masses; the pion mass is about $220 \mathrm{MeV}$ and the strange quark mass is adjusted to its physical value. We have scanned a temperature range approximately from $170 \mathrm{MeV}$ to $500 \mathrm{MeV}$. We are using the exact RHMC algorithm [4] to update configurations. Details on our simulation parameters can be found in [5].

\section{Taylor expansions of thermodynamic quantities}

For a large homogeneous system, the pressure of QCD with $u, d$ and $s$ quarks can be expressed as

$$
\frac{p}{T^{4}}=\frac{1}{V T^{3}} \ln Z\left(V, T, \mu_{u}, \mu_{d}, \mu_{s}\right),
$$

where the partition function $Z$ is a function of the volume $V$, temperature $T$ and chemical potentials of $u, d$, and $s$ quarks. We have not considered other species of quarks whose masses are much heavier. Due to the sign problem, the difficulty of a direct lattice calculation at non zero chemical potentials arises. We perform a Taylor expansion in terms of the chemical potentials

$$
\frac{p}{T^{4}}=\sum_{i, j, k} c_{i j k}(T)\left(\frac{\mu_{u}}{T}\right)^{i}\left(\frac{\mu_{d}}{T}\right)^{j}\left(\frac{\mu_{s}}{T}\right)^{k},
$$

and compute the coefficients $c_{i j k}$ at zero chemical potentials. When the sum $i+j+k$ is odd, the coefficient $c_{i j k}$ is given as expectation value of purely imaginary operators and therefore vanishes exactly. This reflects the invariance of the QCD partition function under change of particle and antiparticle. The leading term $c_{000}$ gives the pressure at vanishing baryon density and can be calculated via the integral method. Results for the parameter values considered here have been presented in [5]. In this work, we will concentrate on the part of the pressure

$$
\Delta p=p(\vec{\mu})-p(\vec{\mu}=0),
$$

that arises due to non-zero chemical potentials, where $\vec{\mu}=\left(\mu_{u}, \mu_{d}, \mu_{s}\right)$. For $i+j+k>0$, the coefficients

$$
c_{i j k}=\left.\frac{1}{i ! j ! k !} \frac{\partial^{i}}{\partial \hat{\mu}_{u}^{i}} \frac{\partial^{j}}{\partial \hat{\mu}_{d}^{j}} \frac{\partial^{k}}{\partial \hat{\mu}_{s}^{k}}\left(p / T^{4}\right)\right|_{\mu=0},
$$

are derivatives of the partition function, and can be calculated on the lattice, where $\hat{\mu}=\mu / T$. These coefficients provide information about other thermal quantities as well. For example, the strange quark number density expands in chemical potentials as

$$
\frac{n_{s}}{T^{3}}=\sum_{i, j, k}(k+1) c_{i j(k+1)} \hat{\mu}_{u}^{i} \hat{\mu}_{d}^{j} \hat{\mu}_{s}^{k}
$$


and similarly the light up and down quark numbers. We can further consider fluctuations in these quantities.

Alternatively, one can introduce chemical potentials for the conserved quantities baryon number $B$, electric charge $Q$ and strangeness $S$, which are related to $\mu_{u}, \mu_{d}, \mu_{s}$ via

$$
\mu_{u}=\frac{1}{3} \mu_{B}+\frac{2}{3} \mu_{Q}, \quad \mu_{d}=\frac{1}{3} \mu_{B}-\frac{1}{3} \mu_{Q}, \quad \mu_{s}=\frac{1}{3} \mu_{B}-\frac{1}{3} \mu_{Q}-\mu_{S}
$$

and compute e.g. the baryon density as

$$
n_{B}=\frac{1}{3}\left(n_{u}+n_{d}+n_{s}\right)
$$

Then we can study densities and fluctuations in $B, Q$ and $S$.

In the following, we will regard $u$ and $d$ quarks as degenerate and consider $2+1$ flavor QCD. With the definition $\mu_{q} \equiv \mu_{u}=\mu_{d}$ for the light quarks, the coefficients are

$$
c_{i j}^{q s}=\left.\frac{1}{i ! j !} \frac{\partial^{i}}{\partial \hat{\mu}_{q}^{i}} \frac{\partial^{j}}{\partial \hat{\mu}_{s}^{j}}\left(p / T^{4}\right)\right|_{\vec{\mu}=\mathbf{0}},
$$

where the subscripts denote the order of the derivative and the superscripts indicate the corresponding flavors. If not specified, the default superscripts will be $q s$ and will often be left out. It is evident from Eqs. (2.6) that choosing $\mu_{u} \equiv \mu_{d}$ is equivalent to a vanishing electric charge potential $\mu_{Q} \equiv 0$.

Now we discuss how to evaluate these coefficients on the lattice. Inserting Eq. (2.1) into Eq. (2.8) and integrating out the fermion fields in the partition function yields the coefficients as expectation values of operators that contain derivatives of the determinant of the fermion matrix $M$. For example the formula for $c_{20}$ reads

$$
c_{20}=\frac{N_{\tau}}{2 N_{\sigma}^{3}}\left(\frac{n_{f}}{4}\left\langle\frac{\partial^{2} \ln \operatorname{det} M}{\partial \vec{\mu}_{q}^{2}}\right\rangle+\left(\frac{n_{f}}{4}\right)^{2}\left\langle\left(\frac{\partial \ln \operatorname{det} M}{\partial \vec{\mu}_{q}}\right)^{2}\right\rangle\right),
$$

where $N_{\tau}$ and $N_{\sigma}$ are temporal and spacial extent of the lattice, $n_{f}$ is the number of quark flavors in question (here $n_{f}=2$ ), and $\langle\cdots\rangle$ indicates taking the thermal average over the ensemble. On each configuration, derivatives of $\ln \operatorname{det} M$ need to be evaluated up to the same order as the order of the expansion coefficients. These derivatives lead to the appearances of the inverse fermion matrix $M^{-1}$ inside traces

$$
\begin{aligned}
\frac{\partial \ln \operatorname{det} M}{\partial \mu} & =\operatorname{Tr}\left(M^{-1} \frac{\partial M}{\partial \mu}\right) \\
\frac{\partial^{2} \ln \operatorname{det} M}{\partial \mu^{2}} & =\operatorname{Tr}\left(M^{-1} \frac{\partial^{2} M}{\partial \mu^{2}}\right)-\operatorname{Tr}\left(M^{-1} \frac{\partial M}{\partial \mu} M^{-1} \frac{\partial M}{\partial \mu}\right) .
\end{aligned}
$$

To avoid full matrix inversions, we use the random noise method in estimating such traces. Suppose we have generated a set of $N$ random noise vectors $R^{(a)}, a=1, \ldots, N$, then the trace can be estimated as

$$
\operatorname{Tr}\left(\mathscr{O} M^{-1}\right) \approx \frac{1}{N} \sum_{a=1}^{N} R^{(a)} \mathscr{O} M^{-1} R^{(a)}
$$


where $\mathscr{O}$ is some arbitrary matrix. For each vector $R^{(a)}$ only the linear system $M X=R^{(a)}$ needs to be solved. It is still quite expensive to compute all necessary operators, since a large number of random vectors is needed in order to get a satisfactory accuracy. Also, higher order coefficients are more expensive, because more operators are needed. For the 4th order coefficients one has

$$
\begin{aligned}
c_{40}= & \frac{1}{4 ! N_{\sigma}^{3} N_{\tau}}\left\{\frac{n_{f}}{4}\left\langle\frac{\partial^{4} \ln \operatorname{det} M}{\partial \mu_{q}^{4}}\right\rangle\right. \\
& +4\left(\frac{n_{f}}{4}\right)^{2}\left\langle\frac{\partial^{3} \ln \operatorname{det} M}{\partial \mu_{q}^{3}} \frac{\partial \ln \operatorname{det} M}{\partial \mu_{q}}\right\rangle+3\left(\frac{n_{f}}{4}\right)^{2}\left\langle\left(\frac{\partial^{2} \ln \operatorname{det} M}{\partial \mu_{q}^{2}}\right)^{2}\right\rangle \\
& +6\left(\frac{n_{f}}{4}\right)^{3}\left\langle\frac{\partial^{2} \ln \operatorname{det} M}{\partial \mu_{q}^{2}}\left(\frac{\partial \ln \operatorname{det} M}{\partial \mu_{q}}\right)^{2}\right\rangle+\left(\frac{n_{f}}{4}\right)^{4}\left\langle\left(\frac{\partial \ln \operatorname{det} M}{\partial \mu_{q}}\right)^{4}\right\rangle \\
& \left.-3\left(\frac{n_{f}}{4}\left\langle\frac{\partial^{2} \ln \operatorname{det} M}{\partial \mu_{q}^{2}}\right\rangle+\left(\frac{n_{f}}{4}\right)^{2}\left\langle\left(\frac{\partial \ln \operatorname{det} M}{\partial \mu_{q}}\right)^{2}\right\rangle\right)^{2}\right\}
\end{aligned}
$$

where

$$
\begin{aligned}
\frac{\partial^{3} \ln \operatorname{det} M}{\partial \mu^{3}}= & \operatorname{Tr}\left(M^{-1} \frac{\partial^{3} M}{\partial \mu^{3}}\right)-3 \operatorname{Tr}\left(M^{-1} \frac{\partial M}{\partial \mu} M^{-1} \frac{\partial^{2} M}{\partial \mu^{2}}\right) \\
& +2 \operatorname{Tr}\left(M^{-1} \frac{\partial M}{\partial \mu} M^{-1} \frac{\partial M}{\partial \mu} M^{-1} \frac{\partial M}{\partial \mu}\right), \\
\frac{\partial^{4} \ln \operatorname{det} M}{\partial \mu^{4}}= & \operatorname{Tr}\left(M^{-1} \frac{\partial^{4} M}{\partial \mu^{4}}\right)-4 \operatorname{Tr}\left(M^{-1} \frac{\partial M}{\partial \mu} M^{-1} \frac{\partial^{3} M}{\partial \mu^{3}}\right) \\
- & 3 \operatorname{Tr}\left(M^{-1} \frac{\partial^{2} M}{\partial \mu^{2}} M^{-1} \frac{\partial^{2} M}{\partial \mu^{2}}\right)+12 \operatorname{Tr}\left(M^{-1} \frac{\partial M}{\partial \mu} M^{-1} \frac{\partial M}{\partial \mu} M^{-1} \frac{\partial^{2} M}{\partial \mu^{2}}\right) \\
- & 6 \operatorname{Tr}\left(M^{-1} \frac{\partial M}{\partial \mu} M^{-1} \frac{\partial M}{\partial \mu} M^{-1} \frac{\partial M}{\partial \mu} M^{-1} \frac{\partial M}{\partial \mu}\right) .
\end{aligned}
$$

Five matrix inversions per random vector are necessary here, while for the 6th order, 12 matrix inversions are needed. Depending on quark mass, temperature and particular operator, different numbers of random vectors are needed to obtain that the errors arising from the stochastic estimator are smaller than or of the same magnitude as the statistical fluctuations within the ensemble.

\section{Pressure and densities}

In this section, we will first show results for the coefficients, then use them in computing pressure and quark number densities.

In Fig. 1 , we show the coefficients $c_{200}$ and $c_{004}$ on both $N_{\tau}=4$ and 6 lattices. $c_{200}$, also known as the fluctuation in $u(d)$ quark number density, increase rapidly through the phase transition region. As one can see, the lattice cut-off effect is small and seems to be under control. Results for $c_{002}$ from $N_{\tau}=8$ lattices [6] further support this statement. The fourth order coefficient $c_{4}$ shows a pronounced peak around $T_{c}$. To compare the quark mass dependence, $c_{200}$ and $c_{002}$ for $u$ and $s$ quarks respectively are shown in Fig. 2. The slope is steeper for light than for the strange quarks, which indicates a stronger sensitivity to the chiral transition for lighter quark masses. We also show 

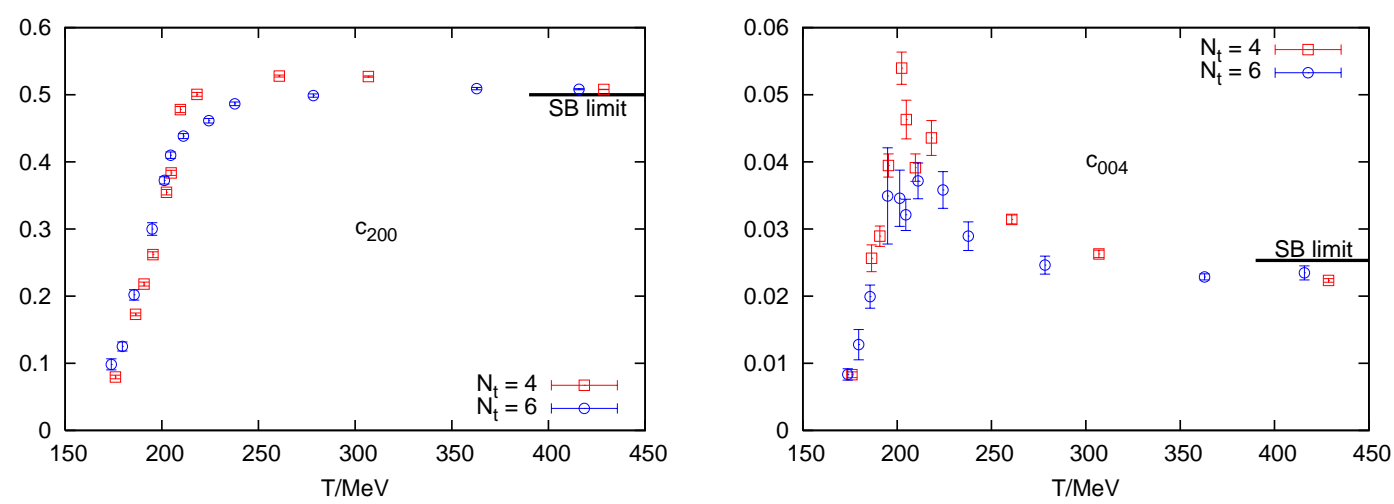

Figure 1: $c_{200}$ on the left and $c_{004}$ on the right for $N_{\tau}=4$ and 6 . The second order coefficients increase rapidly from confined phase to deconfined phase at around $200 \mathrm{MeV}$, while the fourth order ones develop a peak there. Stephen-Boltzmann limits of the free case for the action that we use are marked for both quantities and matched very well in the high temperature region.

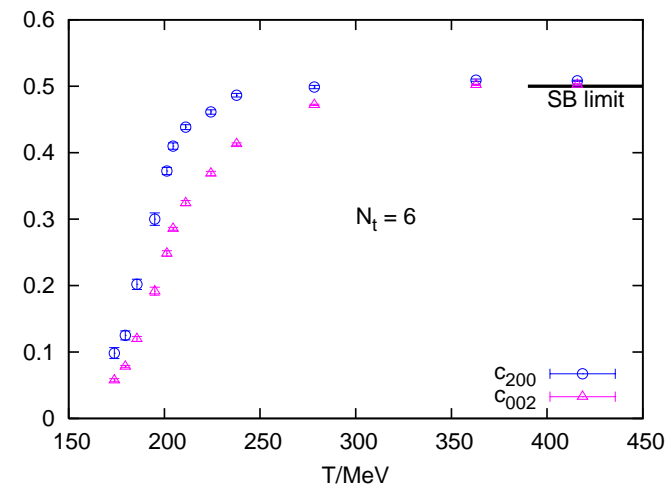

Figure 2: Second order coefficients $c_{200}$ and $c_{002}$ for $u$ and $s$ quark respectively on $N_{\tau}=6$ lattice.

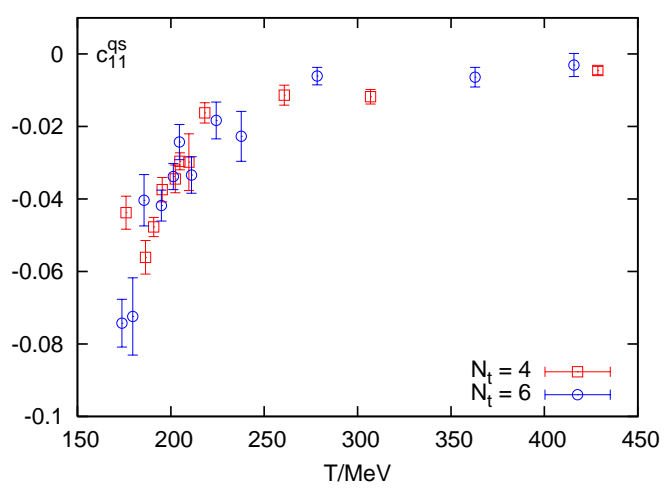

Figure 3: $c_{11}^{q s}$ on $N_{\tau}=4$ and 6 lattices.

$c_{11}^{q s}$ in Fig. 3, which approaches zero from below in the high temperature limit.

Combining all the measured coefficients, we obtain pressure and number density according to formula (2.2) and (2.5). In Fig. 4, we show the pressure difference $\Delta p / T^{4}$ and light quark number density $n_{q} / T^{3}$ at finite light quark chemical potential but zero strange quark chemical potential $\mu_{s}=0$, up to the 4 th order. This should be compared to the pressure at vanishing chemical potential [5], which rises rapidly to a value of about $p / T^{4} \approx 14$ above the transition. The finite density contribution to the pressure adds to this less than $10 \%$ for $\mu_{q} / T<1$.

\section{Hadronic fluctuations at zero and non-zero chemical potential}

Fluctuations of charge densities $n_{B, S, Q}$ are related by the fluctuation dissipation theorem to the second derivatives of the partition function with respect to the corresponding chemical potentials $\mu_{B, S, Q}$. Here $B, S, Q$ denote baryon number, strangeness and electrical charge, respectively. Using Eqs. (2.6) we can rearrange the expansion coefficients $c_{i j k}^{u d s}$ of the pressure to get the coefficients of 

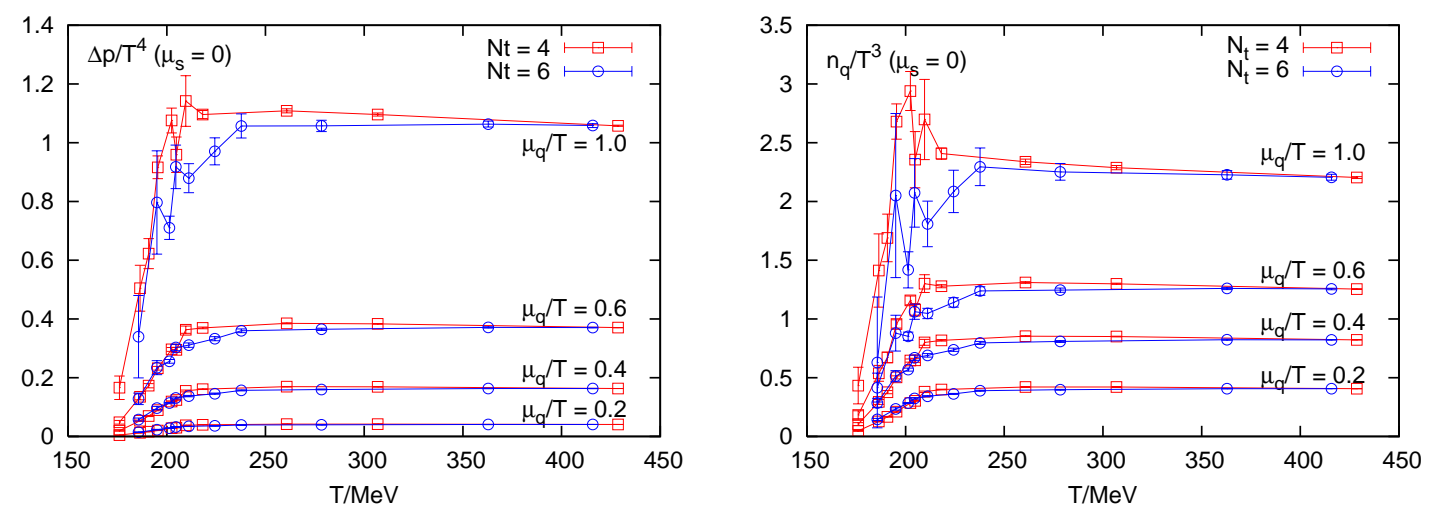

Figure 4: Pressure $\Delta p / T^{4}$ and light quark number density $n_{q} / T^{3}$ at $\mu_{s}=0$ and $\mu_{q} / T=0.2,0.4,0.6$ and 1.0. Small differences are observed between $N_{\tau}=4$ and 6 , especially when $\mu_{q} / T$ is small. Light quark number density seems to develop a peak around $200 \mathrm{MeV}$ when $\mu_{q} / T$ increases.
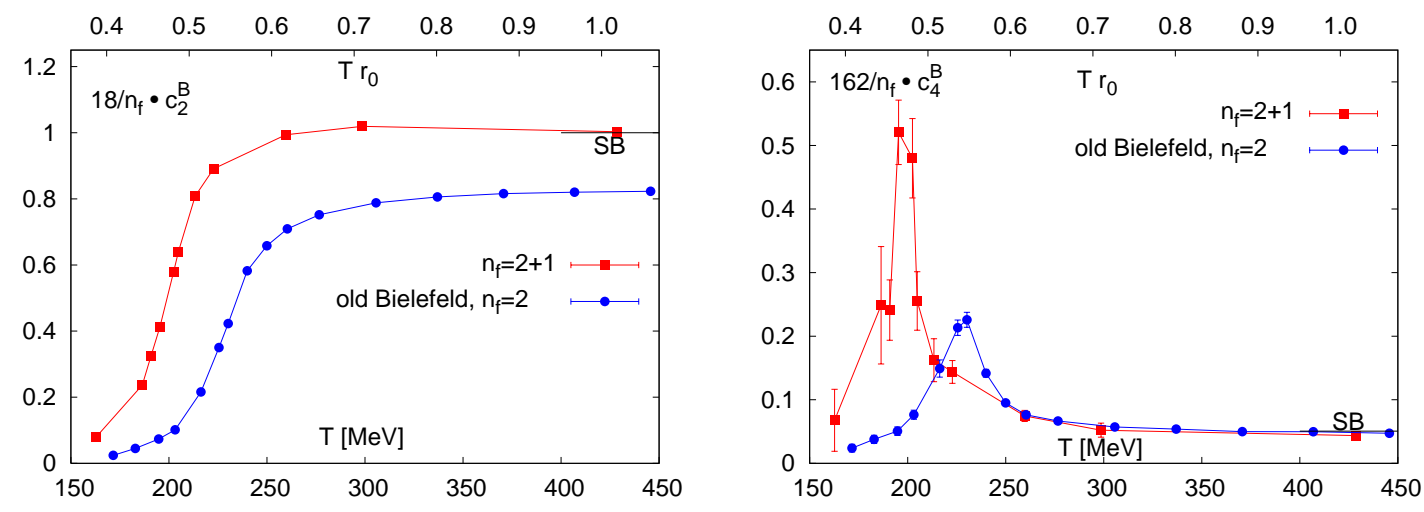

Figure 5: Quadratic and quartic baryon number fluctuations at vanishing net density as function of temperature. Preliminary data from (2+1)-flavor simulations with almost realistic quark masses are compared with previous 2-flavor simulations [2]. Both results have been obtained on $16^{3} \times 4$ lattices.

an expansion in $\mu_{B, S, Q}$, defined as

$$
\frac{p}{T^{4}}=\sum_{i, j, k} c_{i j k}^{B S Q}(T)\left(\frac{\mu_{B}}{T}\right)^{i}\left(\frac{\mu_{S}}{T}\right)^{j}\left(\frac{\mu_{Q}}{T}\right)^{k}
$$

E.g., the following two relations hold for $c_{200}^{B S Q} \equiv c_{2}^{B}$ and $c_{400}^{B S Q} \equiv c_{4}^{B}$

$$
c_{2}^{B}=\frac{1}{9}\left(c_{20}^{q s}+c_{11}^{q s}+c_{02}^{q s}\right), \quad c_{4}^{B}=\frac{1}{81}\left(c_{40}^{q s}+c_{31}^{q s}+c_{22}^{q s}+c_{13}^{q s}+c_{04}^{q s}\right) .
$$

In Fig. 5 we show the first two diagonal expansion coefficients in $\mu_{B} / T$ as function of temperature, which can also be interpreted as the quadratic and quartic baryon number fluctuations. We compare our preliminary results for (2+1)-flavor and almost realistic quark masses to earlier results with 2-flavor and a pion mass $m_{\pi} \approx 700 \mathrm{MeV}$ [2]. The normalization is such that in both cases the same Stefan-Boltzmann value for large temperatures is reached, i.e. we have divided by the number of flavors. An obvious shift in the curves reflects the shift in the transition temperature from 

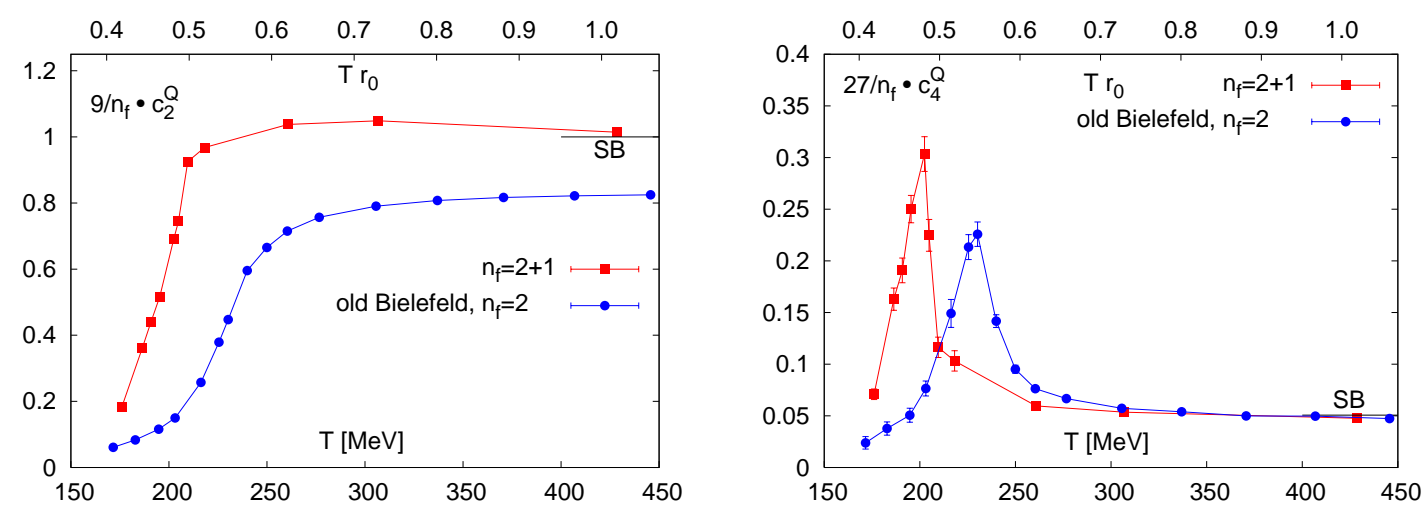

Figure 6: Quadratic and quartic electric fluctuations at vanishing net density as function of temperature. Preliminary data from $(2+1)$-flavor simulations with almost realistic quark masses are compared with previous 2-flavor simulations [2]. Both results have been obtained on $16^{3} \times 4$ lattices.

about $220 \mathrm{MeV}$ to $200 \mathrm{MeV}$. Moreover the sudden change in the quadratic fluctuations is more pronounced for the smaller masses and the Stefan-Boltzmann value is reached faster. Correspondingly, the peak in the quartic fluctuations is higher for smaller masses.

The expansion coefficients in $\mu_{S} / T$ are identical to that in $\mu_{s} / T$ - although the strangeness chemical potential differs from the strange quark chemical potential by a different sign - and are shown in Fig. 1 and 2. In Fig. 6 we show the first two diagonal expansion coefficients in $\mu_{Q} / T$. The qualitative picture is very similar to $\mu_{B} / T$ although the quark mass dependence of the peak height is significantly weaker.

Using the expansion coefficients in $\mu_{B, S, Q} / T$, one can construct hadronic fluctuations at nonzero baryon number density. Up to fourth order correction in $\mu_{B} / T$ we have the following relations for baryon number, strangeness and electric charge fluctuations $\chi_{B S Q}$,

$$
\begin{aligned}
& \frac{\chi_{B}\left(\mu_{B} / T\right)}{T^{2}}=2 c_{2}^{B}+12 c_{4}^{B}\left(\frac{\mu_{B}}{T}\right)^{2}+\mathscr{O}\left[\left(\frac{\mu_{B}}{T}\right)^{4}\right] \\
& \frac{\chi_{S}\left(\mu_{B} / T\right)}{T^{2}}=2 c_{2}^{S}+2 c_{22}^{B S}\left(\frac{\mu_{B}}{T}\right)^{2}+\mathscr{O}\left[\left(\frac{\mu_{B}}{T}\right)^{4}\right] \\
& \frac{\chi_{Q}\left(\mu_{B} / T\right)}{T^{2}}=2 c_{2}^{Q}+2 c_{22}^{B Q}\left(\frac{\mu_{B}}{T}\right)^{2}+\mathscr{O}\left[\left(\frac{\mu_{B}}{T}\right)^{4}\right] .
\end{aligned}
$$

In Fig. 7 we show baryon number and strangeness fluctuations at finite baryon number density. It is obvious that both quantities are developing a peak for increasing $\mu_{B} / T$. However, the peak in $\chi_{B}$ is much more pronounced since this quantity eventually diverges at the critical point in the $\left(T-\mu_{B}\right)$-plane. As we anticipated from Fig. 5, the peak height in $\chi_{B}$ is about twice as large as in earlier calculations with larger quark masses [2]. Note that higher order corrections are still important, especially the position of the peak will be $\mu_{B}$-dependent only by including the next higher order. This has to be analyzed in more detail and eventually will allow to limit the range of values for $\mu_{B} / T$ where the leading order result is reliable.

The off-diagonal coefficients in Eq. 4.1 are usually connected to correlations between baryon number, strangeness and electrical charge. The correlation of baryon number and strangeness can 

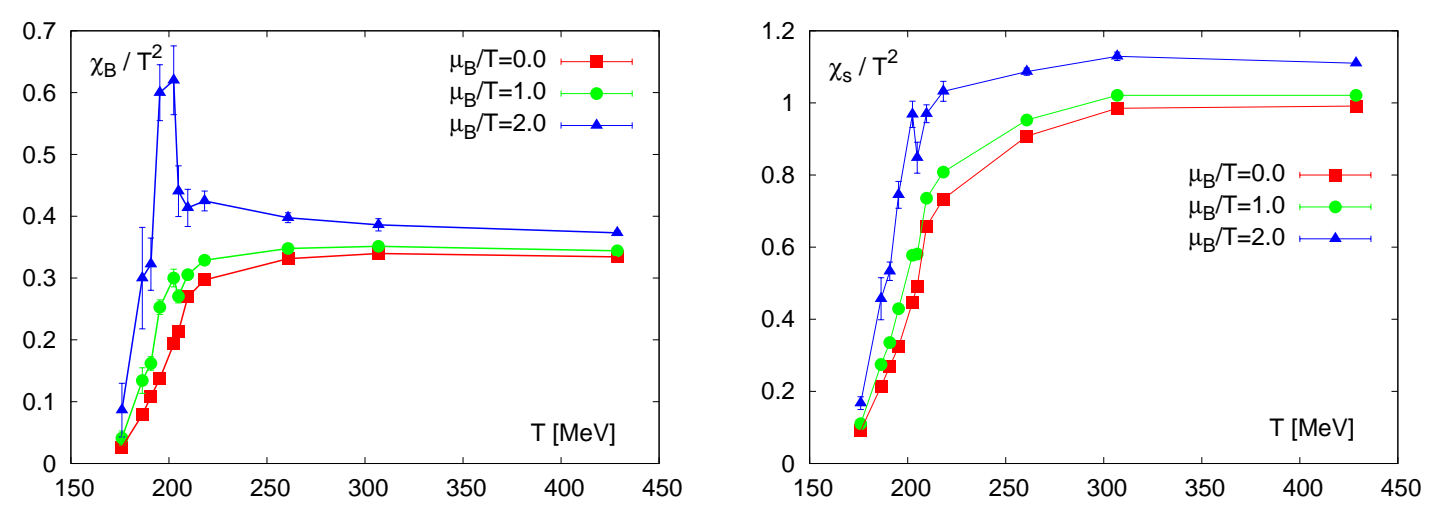

Figure 7: Baryon number and strangeness fluctuations at finite baryon number density, controlled by a finite baryon chemical potential. Results are correct up to fourth order corrections in chemical potential and have been obtained on $16^{3} \times 4$ lattices.
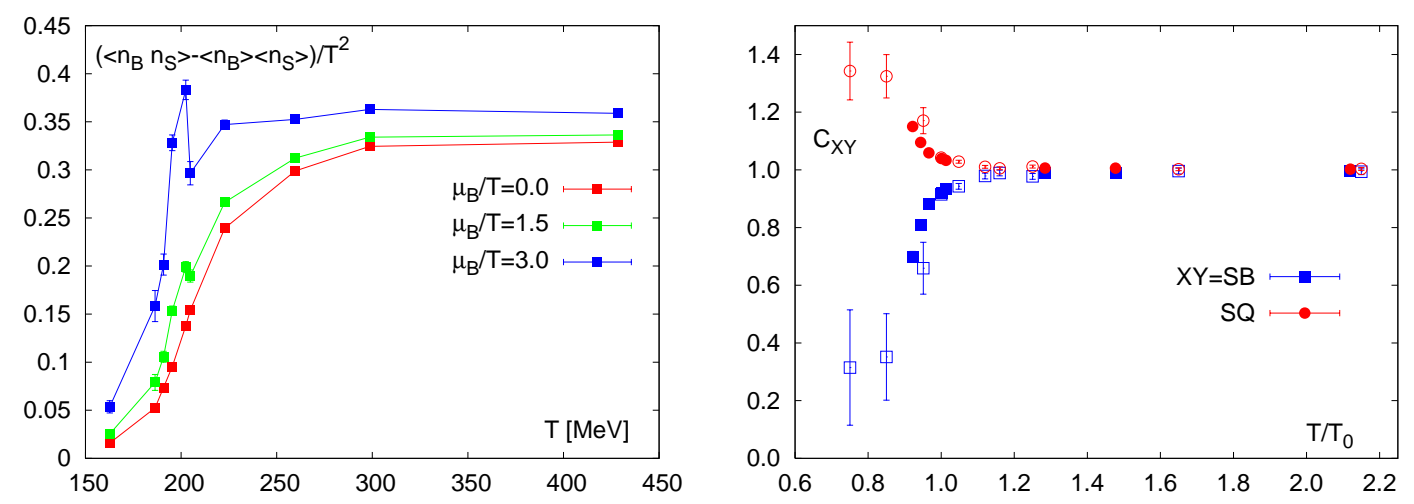

Figure 8: Correlation between baryon number and strangeness for several values of the baryon chemical potential from $16^{3} \times 4$ lattices (left) and the linkage between baryon number and electric charge with strangeness respectively (right). On the right panel we compare or preliminary data (full symbols) to previously obtained results from partially quenched calculations (open symbols) [7], both obtained on $N_{\tau}=4$ lattices.

be expressed in terms of expansion coefficients as

$$
\frac{1}{T^{2}}\left(\left\langle n_{B} n_{S}\right\rangle-\left\langle n_{B}\right\rangle\left\langle n_{S}\right\rangle\right)=c_{11}^{B S}+3 c_{31}^{B S}\left(\frac{\mu_{B}}{T}\right)^{2}+\mathscr{O}\left[\left(\frac{\mu_{B}}{T}\right)^{4}\right]
$$

and is shown in Fig. 8. We find that also this quantity is developing a peak for increasing chemical potential, thus the enhanced correlations suggest the vicinity of a critical point. Another interesting quantity is the "linkage" of strangeness and baryon number or electric charge [7], which is defined as $C_{S X}=c_{11}^{S X} / c_{2}^{S}$, where $X=B, Q$. It is known to be a robust quantity, i.e. the cut-off effects are small. In Fig. 8 (right) we compare our preliminary results with almost realistic quark masses with previously obtained partially quenched results and slightly larger light quark masses [7]. The two calculations show good agreement, thus also the quenching and quark mass effects seem to be small in this quantity. Both results on correlation and linkage between the different quantum numbers suggest that the basic charges are carried by quasi-free quark directly above the transition. This 
seems to rule out the existence of bound states as dominant degrees of freedom in this regime [8].

\section{Conditions at heavy ion colliders and constrained densities}

In general, the pressure, or higher derivatives of the partition functions with respect to chemical potentials, are dependent on at least 3 variables $\mu_{u, d, s}$ or equivalently $\mu_{B, S, Q}$. So far we chose $\mu_{B}>0$, while holding $\mu_{S}=\mu_{Q}=0$. To compare with experiment, for instance heavy ion collisions, the chemical potentials might need to be adjusted to meet the conditions of particular event-byevent fluctuation analyzes [9]. A very natural choice of the chemical potentials is to constrain the strange quark density to zero. Due to the existence of non zero off-diagonal coefficients in Eq. 4.1 we find an increasing strangeness with increasing $\mu_{B}$, even for $\mu_{S}=0$. In heavy ion experiments the total strangeness is zero. Below we outline a procedure to constrain the net strange quark number density $n_{s}$ to zero, subsequently order by order in our $\mu_{B}$ expansion. The procedure can be easily generalized to constrain other charge densities to arbitrary values. This might be of importance, since experiments are often restricted to certain rapidity windows, which may alter expectation values of charge densities.

We can express the strange quark number density $\left(n_{s}\right)$ in terms of the expansion coefficients of the pressure. Up to the 4 th order, it reads

$$
n_{s}=-n_{S}\left(\hat{\mu}_{B}, \hat{\mu}_{S}\right)=-c_{11}^{B S} \hat{\mu}_{B}-2 c_{02}^{B S} \hat{\mu}_{S}-c_{31}^{B S} \hat{\mu}_{B}^{3}-2 c_{22}^{B S} \hat{\mu}_{B}^{2} \hat{\mu}_{S}-3 c_{13}^{B S} \hat{\mu}_{B} \hat{\mu}_{S}^{2}-4 c_{04}^{B S} \hat{\mu}_{S}^{3} \equiv 0,
$$

where $\hat{\mu}=\mu / T$, which means that the strangeness chemical potential $\mu_{S}$ is no longer a free parameter but depends on $\mu_{B}$,

$$
\hat{\mu}_{S}\left(\hat{\mu}_{B}\right)=\left(-\frac{c_{11}^{B S}}{2 c_{02}^{B S}}\right) \hat{\mu}_{B}+\left(\frac{2 c_{04}^{B S} c_{11}^{B S^{3}}-3 c_{02}^{B S} c_{11}^{B S^{2}} c_{13}^{B S}+4 c_{02}^{B S^{2}} c_{11}^{B S} c_{22}^{B S}-4 c_{02}^{B S^{3}} c_{31}^{B S}}{8 c_{02}^{B S^{4}}}\right) \hat{\mu}_{B}^{3}+\mathscr{O}\left(\hat{\mu}_{B}^{5}\right) .
$$

Therefore, the formula for the pressure is modified to

$$
\frac{\Delta p}{T^{4}}=\left(c_{20}^{B S}-\frac{c_{11}^{B S^{2}}}{4 c_{02}^{B S}}\right) \hat{\mu}_{B}^{2}+\left(c_{40}^{B S}+\frac{c_{04}^{B S} c_{11}^{B S^{4}}}{16 c_{02}^{B S^{4}}}-\frac{c_{11}^{B S^{3}} c_{13}^{B S}}{8 c_{02}^{B S}}+\frac{c_{11}^{B S^{2}} c_{22}^{B S}}{4 c_{02}^{B S^{2}}}-\frac{c_{11}^{B S} c_{31}^{B S}}{2 c_{02}^{B S}}\right) \hat{\mu}_{B}^{4}+\mathscr{O}\left(\hat{\mu}_{B}^{6}\right)
$$

which contains off-diagonal coefficients $c_{11}, c_{13}$, etc. On the quark level those coefficients are generally small numbers since they are not present in the free theory. However, on the hadronic level they contain the diagonal strange quark coefficients which have - at least in leading order - a non-zero Stefan-Boltzmann limit. Hence the constraints $n_{S}=0$ and $\mu_{S}=0$ lead to a quite different dependence of the pressure on $\mu_{B} / T$, as can be seen in Fig.9 (left). The difference is almost negligible, when performing an expansion in the light quark chemical potential $\mu_{q} / T$ instead. It is interesting to mention that with the constraint $n_{s}=0$, the pressure expansion in $\mu_{q} / T$ and $\mu_{B} / T$ are identical up to a trivial factor between the two chemical potentials, i.e. the relation $\mu_{B}=3 \mu_{q}$ holds in this case and we have

$$
\Delta p /\left.T^{4}\left(\mu_{q} / T\right)\right|_{n_{s}=0} \equiv \Delta p /\left.T^{4}\left(\mu_{B} / T, \mu_{Q}=0\right)\right|_{n_{S}=0} .
$$

We have also computed the constrained baryon number fluctuations at finite baryon chemical potential $\hat{\chi}_{B}$. Qualitatively, the two cases of $\mu_{S}=0$ and $n_{S}=0$ are very similar. However, it is 

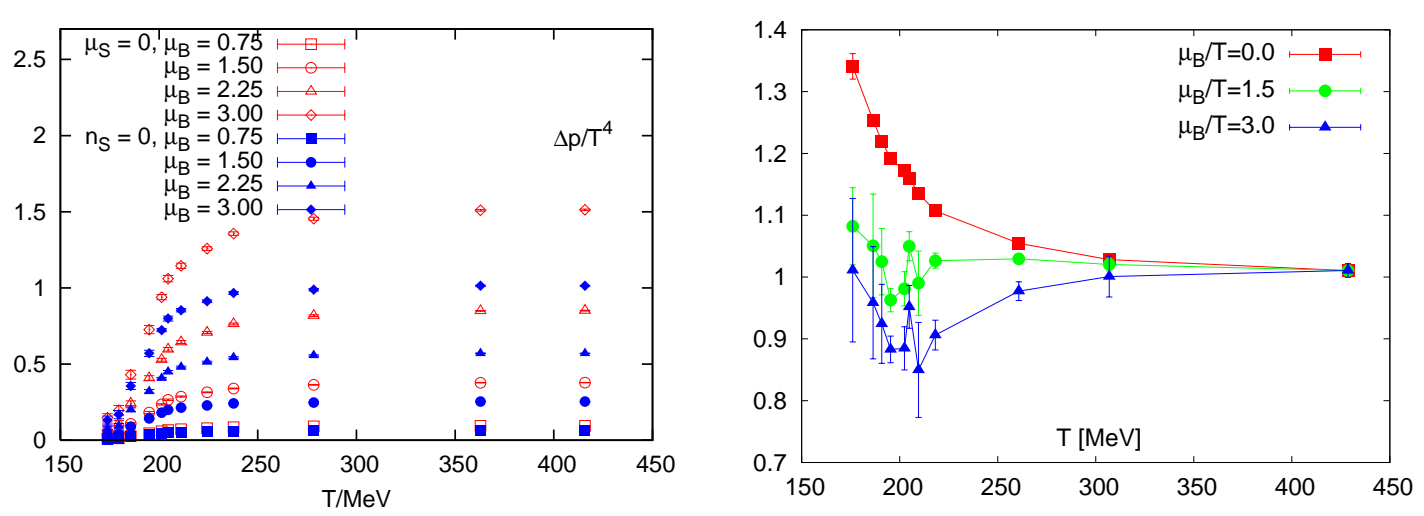

Figure 9: The pressure $\Delta p / T^{4}$ up to the second order for both constraints as labeled (left) and the ratio $\mathscr{N} \hat{\chi}_{B} / \chi_{B}$ as explained in the text (right) for various values of $\mu_{B} / T$. The differences between the two constraints are of the order of $30 \%$ for both quantities. Results have been obtained on $24^{3} \times 6$ lattices (left) and $16^{3} \times 4$ lattices (right).

interesting to remark that the two cases reach different Stefan-Boltzmann limits for high temperatures $(T \rightarrow \infty)$. Taking this into account we show in Fig. 9 (right) the ratio $\mathscr{N} \hat{\chi}_{B} / \chi_{B}$, where $\mathscr{N}$ is the ratio of the corresponding Stefan-Boltzmann values. As one can see, the difference below $T_{C}$ is as high as $30 \%$.

\section{Summary and conclusions}

We have presented a method to rigorously compute corrections to bulk thermodynamic quantities at non vanishing chemical potential, by performing a Taylor expansion in $\mu / T$. Our new preliminary results improved previous calculations in many ways: we went to smaller quark masses, finer lattice spacings and $2+1$ dynamical quark flavor. We also showed how to calculate various hadronic fluctuations, starting from a theory which naturally is formulated in terms of quark fields, as QCD is. The Taylor expansion method provides a variety of input to heavy ion phenomenology.

Our findings are that the finite chemical potential contribution to the pressure is blow $10 \%$, up to a chemical potential of $\mu_{B} / T<3$ and that various hadronic fluctuations develop a peak with increasing baryon chemical potential. This seems to hold true also for strangeness fluctuations, although the peak is much less pronounced in this case. Correlations between strangeness and other charges increase as well when approaching the critical point.

\section{Acknowledgments}

We would like to thank all members of the RBC-Bielefeld Collaboration for helpful discussions and comments. The work has been supported in parts by the U.S. Department of Energy under Contract No. DE-AC02-98CH10886 and by the Deutsche Forschungsgemeinschaft under grant GRK 881. Numerical simulations have been performed on the QCDOC computer of the RIKEN-BNL research center the DOE funded QCDOC at BNL and the APEnext at Bielefeld University. 


\section{References}

[1] R. V. Gavai and S. Gupta, Quark number susceptibilities, strangeness and dynamical confinement, Phys. Rev. D 64 (2001) 074506 [arXiv: hep-lat/0103013];

C. R. Allton, S. Ejiri, S. J. Hands, O. Kaczmarek, F. Karsch, E. Laermann and C. Schmidt, The equation of state for two flavor QCD at non-zero chemical potential, Phys. Rev. D 68 (2003) 014507 [arXiv: hep-lat/0305007];

C. Bernard et al., QCD thermodynamics with $2+1$ flavors at nonzero chemical potential, arXiv:0710.1330 [hep-lat].

[2] C. R. Allton et al., Thermodynamics of two flavor QCD to sixth order in quark chemical potential, Phys. Rev. D 71 (2005) 054508 [hep-lat/0501030].

[3] F. Karsch, E. Laermann and A. Peikert, Quark mass and flavor dependence of the QCD phase transition, Nucl. Phys. B 605 (2001) 579 [hep-lat/ 0012023 ].

[4] A. D. Kennedy, I. Horvath and S. Sint, A new exact method for dynamical fermion computations with non-local actions, Nucl. Phys. Proc. Suppl. 73, 834 (1999) [arXiv: hep-lat/9809092]; M. A. Clark, A. D. Kennedy and Z. Sroczynski, Exact 2+1 flavour RHMC simulations, Nucl. Phys. Proc. Suppl. 140 (2005) 835 [arXiv: hep-lat/ 0409133 ].

[5] M. Cheng et al., The QCD Equation of State with almost Physical Quark Masses, arXiv:0710.0354 [hep-lat] ; J. van der Heide, PoS(LATTICE 2007) 234.

[6] C. DeTar and R. Gupta, PoS(LATTICE 2007) 179; F. Karsch, PoS(LATTICE 2007) 015.

[7] R. V. Gavai and S. Gupta, Fluctuations, strangeness and quasi-quarks in heavy-ion collisions from lattice QCD, Phys. Rev. D 73 (2006) 014004 [arXiv : hep-lat/ 051004 4].

[8] E. V. Shuryak and I. Zahed, Towards a theory of binary bound states in the quark gluon plasma, Phys. Rev. D 70, 054507 (2004) [arXiv: hep-ph/0403127].

[9] V. V. Begun, M. Gazdzicki, M. I. Gorenstein, M. Hauer, V. P. Konchakovski and B. Lungwitz, Multiplicity fluctuations in relativistic nuclear collisions: statistical model versus experimental data, Phys. Rev. C 76 (2007) 024902 [arXiv: nucl-th/0611075]. 